\section{Condições socioeconômicas, oferta de médicos e internações por condições sensíveis à atenção primária em grandes municípios do Brasil}

\author{
Socioeconomic conditions, physician supply, \\ and ambulatory care sensitive hospitalization \\ in large Brazilian cities
}

\section{Condiciones socioeconómicas, oferta de médicos y hospitalizaciones, por condiciones sensibles en la atención primaria, dentro de las grandes ciudades brasileñas}

Ana Luisa Barros de Castro 1 Carla Lourenço Tavares de Andrade 1 Cristiani Vieira Machado 1 Luciana Dias de Lima 1
${ }^{1}$ Escola Nacional de Saúde Pública Sergio Arouca, Fundação Oswaldo Cruz, Rio de Janeiro, Brasil.

Correspondência A. L. B. Castro

Rua Teixeira de Azevedo 93 apto. 1204, bloco A, Rio de Janeiro, RJ 20755-260, Brasil. analuisasb@yahoo.com.br

\begin{abstract}
Ambulatory care sensitive hospitalizations have been used as an indicator of the effectiveness of primary health care. The research in volved a descriptive analysis of the evolution of national indicators from 1998 to 2012 and a cross-sectional study of Brazilian municipalities with populations greater than 50,000, by region of the country, for the year 2012, using correlation and linear regression statistical techniques. There was a slight decline in the proportion of ambulatory care sensitive hospitalizations in Brazil. Socioeconomic and demographic factors and physician supply in the healthcare system are associated with the proportion of ambulatory care sensitive hospitalizations, differing by region of the country. Despite advances in the expansion of the Family Health Strategy, some challenges remain, including better distribution of physicians and other health professionals in the country and effective changes in the healthcare model.
\end{abstract}

Primary Health Care; Hospitalization; Health Services; Family Health Strategy

\section{Resumo}

As internações por condições sensíveis à atenção primária têm sido utilizadas como indicador de efetividade desse nível de atenção. $O$ artigo explora a associação entre variáveis selecionadas e a proporção dessas internações no Brasil. A pesquisa envolveu a análise descritiva da evolução de indicadores nacionais de 1998-2012 e a realização de estudo transversal dos municípios brasileiros com população acima de 50 mil habitantes, por região do país, para o ano de 2012, utilizando-se técnicas estatísticas de correlação e regressão linear. Os resultados mostraram discreto declínio na proporção de internações por condições sensíveis à atenção primária no Brasil. Condições socioeconômicas, demográficas e de oferta de médicos no sistema de saúde nos municípios mostraram associação com a proporção de internações por condições sensiveis à atenção primária, com expressão distinta nas cinco regiões do país. Apesar de avanços relacionados à expansão da Estratégia Saúde da Família, permanecem desafios, como a distribuição adequada de médicos e outros profissionais no território nacional e a efetiva mudança do modelo de atenção à saúde.

Atenção Primária à Saúde; Hospitalização; Serviços de Saúde; Estratégia Saúde da Família 


\section{Introdução}

Nas últimas décadas, o debate sobre o papel e a importância da atenção primária à saúde (APS) na organização dos sistemas ganhou destaque internacionalmente 1,2,3. Diferentes estudos reconhecem vantagens nos sistemas de saúde centrados na atenção primária em relação aos baseados em cuidados especializados, com destaque para questões referentes à equidade, ao acesso, ao menor custo, à continuidade do cuidado, bem como melhorias nos indicadores de saúde da população 4,5 .

Tal debate é permeado por diferentes concepções de APS. A perspectiva abrangente aponta a APS como componente fundamental de um sistema de saúde eficaz. Já a concepção seletiva refere-se a um pacote de intervenções técnicas de baixo custo para combater as principais doenças que acometem as populações pobres, especialmente nos países em desenvolvimento 6 .

As concepções de atenção primária variam nos diferentes sistemas de saúde em função das características gerais da proteção social - mais universalista ou focalizada - e das particularidades da trajetória das políticas de saúde, conformadas em diferentes contextos sociais, políticos e econômicos, sob interesses conflitantes. Nas décadas de 1980 e 1990, a concepção de atenção primária seletiva predominou nos processos de reforma dos sistemas de saúde na maior parte dos países latino-americanos 7,8. Nos anos 2000, a Organização Mundial da Saúde (OMS) e a Organização Pan-Americana da Saúde (OPAS) reconheceram os limites da proposta seletiva e lançaram a estratégia designada como Renovação da Atenção Primária à Saúde nas Américas 9 , em defesa da adoção de uma perspectiva abrangente de APS, ainda que adaptável ao contexto dos distintos países 2,9 .

O reconhecimento da relevância da organização dos sistemas de saúde a partir da atenção primária também impulsionou o desenvolvimento de propostas de avaliação de sua efetividade em termos da redução da morbidade e da mortalidade por diferentes condições. Ainda nos anos 1990, Billings et al. 10 propuseram o termo condições sensíveis à atenção primária (ambulatory care sensitive conditions) para designar um conjunto de problemas de saúde para os quais a ação nesse nível de atenção diminuiria o risco de internações, por meio de prevenção de doenças, diagnóstico e tratamento precoce de condições agudas, controle e acompanhamento de patologias crônicas.

Embora as internações por condições sensíveis à atenção primária possuam natureza diversa e sofram influência de fatores como renda, es- colaridade e acesso aos serviços de saúde privados ou especializados 11,12, tal indicador tem sido amplamente utilizado como medida de acesso e efetividade dos cuidados ofertados nesse nível de atenção, compondo a lista de indicadores de qualidade da atenção primária nos paísesmembros da Organização para a Cooperação e Desenvolvimento Econômico (OCDE). Dentre os efeitos deletérios ao sistema de saúde gerados por internações "desnecessárias", relacionadas às condições sensíveis à atenção primária, Carneiro et al. 13 destacam o aumento de iatrogenias e de custos, com repercussão na assistência à saúde de forma global.

As mudanças no perfil demográfico e epidemiológico em países em desenvolvimento, com o crescimento da morbidade e da mortalidade por doenças crônicas não transmissíveis, colocam novos desafios para a organização e a avaliação do desempenho da atenção primária no contexto dos sistemas de saúde.

No Brasil, desde meados da década de 1990, o Governo Federal tem investido na reorganização da APS no país, com ênfase na Estratégia Saúde da Família (ESF), que alcançou cobertura estimada de mais de $56 \%$ da população brasileira em 2013. Essa expansão foi expressiva, porém desigual no país, marcada por um ritmo mais acelerado nos municípios de pequeno e médio porte e dificuldades em grandes centros urbanos, relacionadas às características prévias dos sistemas de saúde e maior presença do setor privado, entre outros fatores 14,15.

Além disso, numerosos estudos apontam limitações no acesso, na continuidade e na qualidade da assistência prestada nesse nível de atenção 16,17,18. Em consequência, a partir dos anos 2000, expandiram-se as iniciativas de monitoramento e avaliação da APS no Brasil.

Nesse contexto, após intenso debate entre especialistas, o Ministério da Saúde propôs a lista brasileira de grupos de causas de internações e diagnósticos considerados condições sensíveis à atenção primária 19 . Vale ressaltar a importância da adaptação da lista ao contexto brasileiro, visto que as características dos sistemas de saúde, os perfis epidemiológicos e a carga de doenças diferem entre países 20 . Além disso, o processo de adaptação é importante para garantir validade, confiabilidade e representatividade da lista, além de ser um pré-requisito fundamental para a utilização.

Diferentes estudos sugerem um impacto positivo da expansão da ESF na redução de internações por condições sensíveis à atenção primária em municípios brasileiros 21,22,23. No entanto, os índices de internações por condições sensíveis à atenção primária no Brasil permanecem eleva- 
dos - em geral, acima de $20 \%$ das internações em comparação a outros países com sistemas de saúde universais, tais como Espanha e Austrália, que apresentam taxas de internações por condições sensíveis à atenção primária em torno de $7 \%$ a $13 \%$, respectivamente 24 .

Nesse sentido, a pesquisa apresentada no artigo foi orientada pela seguinte questão: que fatores estão associados à ocorrência de internações por condições sensíveis à atenção primária no Brasil? Partiu-se da hipótese geral de que a ocorrência dessas internações pode ser influenciada por distintas variáveis, como condições socioeconômicas, demográficas, de oferta de serviços e de configuração do sistema de saúde. Porém, dada a heterogeneidade territorial do país, essa influência não ocorreria de forma homogênea entre as regiões brasileiras.

Este artigo tem o propósito de apresentar os resultados do estudo que analisou as variáveis associadas à ocorrência de internações por condições sensíveis à atenção primária em saúde nos municípios brasileiros com população acima de 50 mil habitantes, separadamente pelas cinco grandes regiões do país.

\section{Método}

No desenho do estudo, definiu-se, como variável dependente (ou desfecho), a proporção de internações por condições sensíveis à atenção primária no âmbito do Sistema Único de Saúde (SUS). Já as condições que poderiam expressar associações com o desfecho foram designadas como variáveis independentes, expressas pelos seguintes indicadores selecionados:

a) Condições socioeconômicas - o indicador Índice de Desenvolvimento Humano Municipal (IDH-M) foi utilizado como proxy;

b) Condições demográficas - o indicador proporção da população com 60 anos ou mais foi selecionado devido ao maior risco de internações entre os idosos;

c) Oferta de serviços de saúde - esse grupo envolveu indicadores relativos ao modelo de atenção básica (cobertura da ESF), à oferta de profissionais médicos, de leitos e de equipamentos diagnósticos. No último caso, foram selecionados os principais equipamentos diagnósticos que, na visão dos autores, seriam necessários para atendimento às pessoas com condições incluídas na lista brasileira de condições sensíveis à atenção primária 19. Embora o mamógrafo não esteja diretamente relacionado às condições presentes na referida lista, tal equipamento também foi incluído no estudo pela sua relevância para diagnóstico do câncer de mama, cujas taxas de incidência e mortalidade permanecem elevadas no Brasil 25;

d) Peso dos planos privados no sistema de saúde - proporção de beneficiários de planos privados de saúde na população, que reflete a importância do setor privado suplementar.

Este estudo foi efetuado em duas etapas, a partir das bases de dados nacionais. A primeira consistiu em uma análise exploratória descritiva em série temporal do Brasil, abrangendo o período de 1998 a 2012. Analisou-se a evolução de parte dos indicadores incluídos no modelo de análise, bem como a variação percentual no período. O ano de 1998 foi selecionado, pois representa o início do registro oficial do número de equipes de saúde da família implantadas pelos municípios por meio do Sistema de Informação da Atenção Básica (SIAB). Utilizou-se o ano de 2012 como ano mais recente, para assegurar a disponibilidade e confiabilidade dos dados. Cabe ressaltar que o IDH-M não estava disponível para os anos de 1998 e 2012. No entanto, pela sua importância como proxy das condições socioeconômicas da população, foram levantados os dados do IDH$\mathrm{M}$ referentes aos anos de 2000 e 2010 . Por outro lado, no que diz respeito à oferta de serviços, foram utilizados nessa etapa somente os indicadores relativos à atenção básica (cobertura da ESF), oferta de médicos e leitos hospitalares. Os indicadores referentes à oferta de equipamentos diagnósticos não foram incluídos por limites de disponibilidade e comparabilidade, visto que o Cadastro Nacional de Estabelecimentos de Saúde (CNES) ainda não havia sido implantado em 1998.

A segunda etapa consistiu na realização de um estudo ecológico, comparando as regiões brasileiras (Norte, Nordeste, Centro-oeste, Sudeste e Sul) no ano de 2012, a partir da construção de uma base de dados dos municípios com população igual ou superior a 50 mil habitantes $(\mathrm{n}=614)$.

A base de dados inicialmente incluiu todas as internações hospitalares realizadas em 2012, segundo município de residência do paciente, registradas pelo Sistema de Informação Hospitalar do SUS (SIH-SUS). Calculou-se a proporção de internações por condições sensíveis à atenção primária (variável desfecho), considerando a lista brasileira de condições sensíveis à atenção primária adotada pelo Ministério da Saúde ${ }^{19}$. A seleção dos municípios com população acima de 50 mil habitantes como unidade de análise do estudo ocorreu após aplicação do teste de Kolmogorov-Smirnov, que avalia se a variável desfecho segue uma distribuição normal, pressuposto para a realização da técnica estatística de regressão linear. Todas as variáveis 
utilizadas na primeira etapa do estudo foram incorporadas nessa fase. A Tabela 1 expõe, de forma sintética, as variáveis e os indicadores incluídos no estudo, suas fórmulas de cálculo e a fonte de dados. Para cálculo dos indicadores, foram utilizadas as estimativas populacionais do Instituto Brasileiro de Geografia e Estatística (IBGE).

Com o intuito de verificar se as variáveis selecionadas estão associadas à proporção de internações por condições sensíveis à atenção primária nos diferentes municípios e regiões brasileiras, foram utilizadas as técnicas estatísticas de correlação e regressão linear. A dependência linear entre as variáveis foi medida pelo coeficiente de correlação de Pearson, que avalia o sentido (positivo ou negativo) e a intensidade da relação entre as variáveis, que foi classificada como fraca $(r \geq 0$ e $\leq 0,3)$, moderada $(r \geq 0,3$ e $\leq$ $0,6)$ ou forte $(r \geq 0,6$ e $\leq 0,9) 26$. Todas as variáveis com associação estatisticamente significativa ( $p$ $\leq 0,05$ ) foram incluídas na análise.

A seguir, foram efetuadas cinco análises de regressão linear múltipla com intuito de identificar quais dentre as variáveis independentes melhor explicam a variação do desfecho em cada região do país.

A construção da base de dados a partir dos dados disponíveis no SIH-SUS foi efetuada com auxílio do programa SAS (SAS Inst., Cary, Estados Unidos). Para cálculo dos indicadores e análise dos dados, foi utilizado o programa IBM SPSS (IBM Corp., Armonk, Estados Unidos). Foram utilizados somente dados de domínio público e acesso irrestrito. O estudo compõe um projeto mais amplo que foi aprovado pelo Comitê de Ética em Pesquisa da Escola Nacional de Saúde Pública Sergio Arouca, Fundação Oswaldo Cruz, parecer no 423.718.

\section{Resultados}

A análise inicial demonstrou que, entre 1998 e 2012, houve uma diminuição da proporção de internações por condições sensíveis à atenção primária de cerca de $15 \%$ no Brasil. No que diz respeito à oferta de serviços, observou-se aumento nacional em mais de $700 \%$ na cobertura populacional pela ESF, enquanto a disponibilidade de médicos por mil habitantes cresceu cerca de $16 \%$ no mesmo período. Chama ainda a atenção a redução de mais de $20 \%$ da disponibilidade de leitos totais e privados no país no período (Tabela 2).

Vale ressaltar que o IDH-M, apesar de não disponível para os anos de 1998 e 2012, apresentou queda expressiva de quase $19 \%$ entre 2000 e
2010, segundo os dados do Programa das Nações Unidas para o Desenvolvimento (PNUD).

A Figura 1 mostra a evolução anual da cobertura pela ESF e da proporção de internações por condições sensíveis à atenção primária, no período de 1998 a 2012. A cobertura pela ESF apresentou tendência ascendente em todo o período. Já em relação à proporção de internações por condições sensíveis à atenção primária, observa-se a manutenção de um padrão estável em patamares elevados, em torno de $24 \%$, nos dez primeiros anos da série, com ligeira queda a partir de 2008, alcançando 20,4\% em 2012.

Os resultados da análise do banco de dados dos 614 municípios agregados por região referentes ao ano de 2012, por meio da utilização das técnicas de correlação de Pearson e da análise de regressão linear, encontram-se expostos nas Tabelas 3 e 4 .

A Tabela 3 mostra que nenhuma das variáveis analisadas mostrou correlação significativa com as internações por condições sensíveis à atenção primária na Região Centro-oeste. Nas demais regiões, o mesmo ocorreu com quatro variáveis: leitos privados por mil habitantes; proporção de médicos SUS; eletrocardiograma (ECG) total por milm habitantes e ultrassonografia total por mil habitantes.

As demais variáveis independentes incluídas no modelo mostraram correlação fraca ou moderada com as internações por condições sensíveis à atenção primária em, ao menos, uma região. Vale destacar que somente as variáveis IDH-M e proporção de beneficiários de planos de saúde apresentaram correlação negativa com a variável desfecho para quatro regiões (Norte, Nordeste, Sudeste e Sul). Outras variáveis que se destacaram ao apresentar correlação significativa para três diferentes regiões foram: proporção de idosos (correlação positiva moderada no Sul, fraca no Nordeste e Sudeste); proporção de leitos SUS (correlação positiva moderada no Norte, fraca no Nordeste e Sudeste); total de médicos por mil habitantes (correlação negativa moderada no Norte, fraca no Nordeste e Sul).

As variáveis relativas à oferta de equipamentos diagnósticos mostraram correlação fraca com as internações por condições sensíveis à atenção primária em apenas uma ou duas regiões.

Já a proporção da população coberta pela ESF apresentou correlação negativa estatisticamente significativa com as internações por condições sensíveis à atenção primária somente na região Sudeste do país, ainda assim classificada como fraca.

A Tabela 4 apresenta os resultados da regressão linear, que foi realizada para as cinco regiões do país. No caso da Região Centro-oeste, assim 
Variáveis e indicadores incluídos no estudo.

\begin{tabular}{|c|c|c|}
\hline Variáveis/Indicadores & Forma de cálculo & Fonte \\
\hline \multicolumn{3}{|l|}{ Variável dependente } \\
\hline \multicolumn{3}{|l|}{ Internações por condições sensíveis à atenção primária } \\
\hline $\begin{array}{l}\text { Proporção de Internações por condições sensiveis à } \\
\text { atenção primária }\end{array}$ & $\begin{array}{c}\text { Número de pessoas internadas por procedimentos } \\
\text { classificados na Portaria no } 221 \text { 19, como sensíveis à atenção } \\
\text { primária/Total de internações x } 100\end{array}$ & SIH-SUS \\
\hline \multicolumn{3}{|l|}{ Variáveis independentes } \\
\hline \multicolumn{3}{|l|}{ Condições socioeconômicas } \\
\hline IDH-M & $3 \sqrt{ }$ IDH-M longevidade $\times$ IDH-M educação $\times$ IDH-M renda & PNUD \\
\hline \multicolumn{3}{|l|}{ Condições demográficas } \\
\hline Proporção da população com 60 anos ou mais & $\begin{array}{l}\text { População com idade igual ou superior a } 60 \text { anos/ população } \\
\qquad \text { total } \times 100\end{array}$ & IBGE \\
\hline \multicolumn{3}{|l|}{ Oferta de serviços de saúde } \\
\hline Proporção da população coberta pela ESF & Estimativa da população coberta pela ESF/população x 100 & $\begin{array}{l}\text { MS/SAS/DAB e } \\
\text { IBGE }\end{array}$ \\
\hline Total de leitos/1.000 habitantes & Leitos de internação existentes/população x 1.000 & $\begin{array}{l}\text { MS/SAS/SIH/SUS } \\
\text { CNES e IBGE }\end{array}$ \\
\hline Proporção de leitos SUS & Leitos SUS/leitos de internação existentes x 100 & $\begin{array}{l}\text { MS/SAS/SIH/SUS } \\
\text { CNES e IBGE }\end{array}$ \\
\hline Leitos privados/1.000 habitantes & Leitos não SUS/população x 1.000 & $\begin{array}{l}\text { MS/SAS/SIH/SUS } \\
\text { CNES e IBGE }\end{array}$ \\
\hline Médicos/1.000 habitantes & $\begin{array}{l}\text { Total de médicos (indivíduos segundo CBO 2002)/população } \\
\qquad \times 1.000\end{array}$ & CFM e IBGE \\
\hline Proporção de médicos SUS & $\begin{array}{l}\text { Total de médicos que atendem no SUS (indivíduos segundo } \\
\qquad \text { CBO 2002)/total de médicos x } 100\end{array}$ & CNES e IBGE \\
\hline ECG/10.000 habitantes & ECG existentes/população x 10.000 & CNES e IBGE \\
\hline ECG disponíveis ao SUS/10.000 habitantes & ECG existentes disponíveis ao SUS/população x 10.000 & CNES e IBGE \\
\hline Endoscópios digestivos/10.000 habitantes & Endoscópios digestivos existentes/população x 10.000 & CNES e IBGE \\
\hline $\begin{array}{l}\text { Endoscópios digestivos disponíveis ao SUS/10.000 } \\
\text { habitantes }\end{array}$ & $\begin{array}{l}\text { Endoscópios digestivos existentes disponíveis ao SUS/ } \\
\qquad \text { população } \times 10.000\end{array}$ & CNES e IBGE \\
\hline Mamógrafos/10.000 habitantes & Mamógrafos existentes/população x 10.000 & CNES e IBGE \\
\hline Mamógrafos disponíveis ao SUS/10.000 habitantes & $\begin{array}{l}\text { Mamógrafos existentes disponíveis ao SUS/população x } \\
10.000\end{array}$ & CNES e IBGE \\
\hline $\begin{array}{l}\text { Equipamentos de radiologia médica total/10.000 } \\
\text { habitantes }\end{array}$ & $\begin{array}{l}\text { Equipamentos de radiologia médica existentes/população x } \\
\qquad 10.000\end{array}$ & CNES e IBGE \\
\hline $\begin{array}{l}\text { Equipamentos de radiologia médica disponíveis ao } \\
\text { SUS/10.000 habitantes }\end{array}$ & $\begin{array}{l}\text { Equipamentos de radiologia médica disponíveis ao SUS/ } \\
\qquad \text { população } \times 10.000\end{array}$ & CNES e IBGE \\
\hline Equipamentos de ultrassonografia/10.000 habitantes & $\begin{array}{l}\text { Equipamentos de ultrassonografia existentes/população x } \\
\qquad 10.000\end{array}$ & CNES e IBGE \\
\hline $\begin{array}{l}\text { Equipamentos de ultrassonografia disponíveis ao } \\
\text { SUS/10.000 habitantes }\end{array}$ & $\begin{array}{l}\text { Equipamentos de ultrassonografia disponíveis ao SUS/ } \\
\qquad \text { população } \times 10.000\end{array}$ & CNES e IBGE \\
\hline \multicolumn{3}{|l|}{ Peso dos planos privados no sistema de saúde } \\
\hline Proporção de beneficiários de planos de saúde & Beneficiários de planos de saúde/população x 100 & ANS e IBGE \\
\hline
\end{tabular}

ANS: Agência Nacional de Saúde Suplementar; CBO: Classificação Brasileira de Ocupações; CFM: Conselho Federal de Medicina; CNES: Cadastro Nacional de Estabelecimentos de Saúde; DAB: Departamento de Atenção Básica; ECG: eletrocardiograma; ESF: Estratégia Saúde da Família; IBGE: Instituto Brasileiro de Geografia e Estatística; IDH-M: Índice de Desenvolvimento Humano Municipal; MS: Ministério da Saúde; PNUD: Programa das Nações Unidas para o Desenvolvimento; SAS: Secretaria de Atenção á Saúde; SIH: Sistema de Informações Hospitalares; SUS: Sistema Único de Saúde. 
Tabela 2

Evolução de indicadores selecionados. Brasil, 1998 e 2012.

\begin{tabular}{|c|c|c|c|}
\hline Indicador & 1998 & 2012 & Variação média \\
\hline & & & 1998-2012 (\%) \\
\hline \multicolumn{4}{|l|}{ Variável dependente } \\
\hline Proporção de internações por condições sensíveis à atenção primária * & 24,1 & 20,4 & $-15,35$ \\
\hline \multicolumn{4}{|l|}{ Variáveis independentes } \\
\hline IDH-M (2000-2010) ** & 0,61 & 0,73 & 18,79 \\
\hline Proporção da população com 60 anos ou mais *** & 7,85 & 10,77 & 37,15 \\
\hline Proporção da população coberta pela ESF *** & 6,55 & 54,84 & 737,25 \\
\hline Total de leitos/1.000 habitantes \#\# & 3,05 & 2,35 & $-22,95$ \\
\hline Proporção de leitos não SUS \#\# & 23,17 & 36,67 & 58,26 \\
\hline Leitos privados/1.000 habitantes \#\# & 2,05 & 1,49 & $-27,32$ \\
\hline Médicos/1.000 habitantes \#\#\# & 1,69 & 1,95 & 15,38 \\
\hline
\end{tabular}

ESF: Estratégia Saúde da Família; IDM-M: Índice de Desenvolvimento Humano municipal; SUS: Sistema Único de Saúde.

* PROADESS 24;

** Programa das Nações Unidas para o Desenvolvimento;

*** Instituto Brasileiro de Geografia e Estatística;

\# Departamento de Atenção Básica, Ministério da Saúde;

\#\# Sistema de Informações Hospitalares/Cadastro Nacional de Estabelecimentos de Saúde/Instituto Brasileiro de Geografia e Estatística;

\#\#\# Conselho Federal de Medicina/Instituto Brasileiro de Geografia e Estatística.

Figura 1

Evolução da cobertura pela Estratégia Saúde da Família e percentual de internações sensíveis à atenção primária. Brasil, 1998-2012.

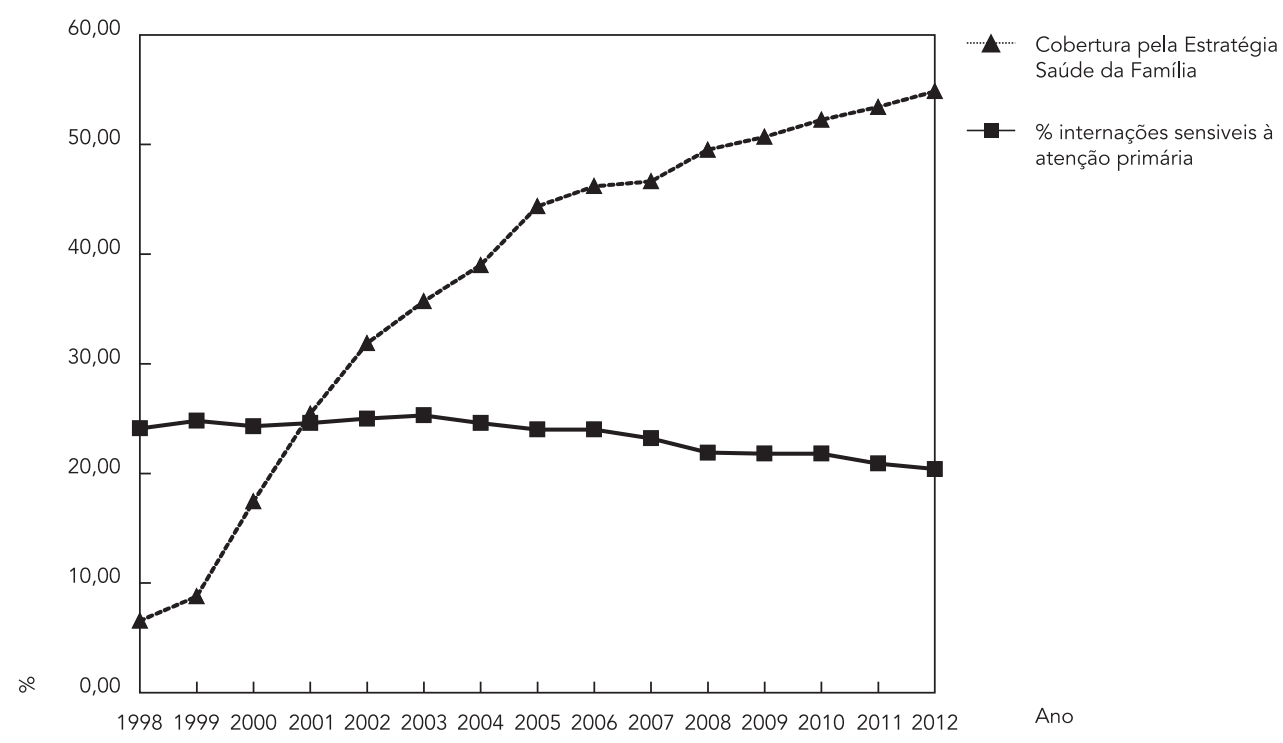


Tabela 3

Coeficiente de correlação de Pearson (r) e valor de p. Correlação entre proporção de internações por condições sensíveis à atenção primária e variáveis associadas nas diferentes regiões brasileiras, 2012.

\begin{tabular}{|c|c|c|c|c|c|}
\hline \multirow[t]{2}{*}{ Variável } & \multicolumn{5}{|c|}{ Regiões } \\
\hline & $\begin{array}{l}\text { Centro-oeste } \\
\qquad(n=35)\end{array}$ & $\begin{array}{c}\text { Norte } \\
(n=63)\end{array}$ & $\begin{array}{l}\text { Nordeste } \\
(n=174)\end{array}$ & $\begin{array}{l}\text { Sudeste } \\
(n=241)\end{array}$ & $\begin{array}{c}\text { Sul } \\
(n=101)\end{array}$ \\
\hline \multicolumn{6}{|l|}{ IDH-M } \\
\hline Correlação de Pearson & $-0,257$ & $-0,354$ & $-0,356$ & $-0,207$ & $-0,426$ \\
\hline Valor de $p$ & 0,136 & 0,004 & 0,000 & 0,001 & 0,000 \\
\hline \multicolumn{6}{|c|}{ Proporção da população coberta pela ESF } \\
\hline Correlação de Pearson & $-0,002$ & $-0,043$ & 0,023 & 0,198 & $-0,089$ \\
\hline Valor de $p$ & 0,990 & 0,740 & 0,763 & 0,002 & 0,374 \\
\hline \multicolumn{6}{|c|}{ Total de leitos/1.000 habitantes } \\
\hline Correlação de Pearson & 0,141 & 0,229 & 0,334 & 0,065 & 0,149 \\
\hline Valor de $p$ & 0,434 & 0,107 & 0,000 & 0,342 & 0,150 \\
\hline \multicolumn{6}{|l|}{ Proporção de leitos SUS } \\
\hline Correlação de Pearson & 0,229 & 0,368 & 0,264 & 0,213 & 0,116 \\
\hline Valo de $\mathrm{p}$ & 0,240 & 0,032 & 0,003 & 0,003 & 0,278 \\
\hline \multicolumn{6}{|c|}{ Leitos privados/1.000 habitantes } \\
\hline Correlação de Pearson & 0,225 & 0,054 & 0,080 & $-0,055$ & 0,018 \\
\hline Valor de $p$ & 0,215 & 0,716 & 0,382 & 0,422 & 0,863 \\
\hline \multicolumn{6}{|l|}{ Médicos/1.000 habitantes } \\
\hline Correlação de Pearson & $-0,224$ & $-0,364$ & $-0,217$ & $-0,067$ & $-0,237$ \\
\hline Valor de $p$ & 0,197 & 0,003 & 0,004 & 0,302 & 0,017 \\
\hline \multicolumn{6}{|l|}{ Proporção de médicos SUS } \\
\hline Correlação de Pearson & $-0,062$ & 0,168 & 0,111 & 0,089 & 0,170 \\
\hline Valor de $p$ & 0,724 & 0,189 & 0,143 & 0,169 & 0,089 \\
\hline \multicolumn{6}{|c|}{ Proporção da população com 60 anos ou mais } \\
\hline Correlação de Pearson & 0,218 & 0,229 & 0,252 & 0,223 & 0,379 \\
\hline Valor de $p$ & 0,209 & 0,072 & 0,001 & 0,000 & 0,000 \\
\hline \multicolumn{6}{|c|}{ Proporção de beneficiários de planos de saúde } \\
\hline Correlação de Pearson & 0,085 & $-0,333$ & $-0,355$ & $-0,237$ & $-0,390$ \\
\hline Valor de $p$ & 0,629 & 0,008 & 0,000 & 0,000 & 0,000 \\
\hline \multicolumn{6}{|l|}{ ECG/10.000 habitantes } \\
\hline Correlação de Pearson & $-0,034$ & $-0,196$ & $-0,025$ & 0,033 & $-0,040$ \\
\hline Valor de $p$ & 0,846 & 0,127 & 0,742 & 0,607 & 0,692 \\
\hline \multicolumn{6}{|c|}{ ECG disponíveis ao SUS/10.000 habitantes } \\
\hline Correlação de Pearson & 0,171 & 0,108 & 0,178 & $-0,014$ & 0,106 \\
\hline Valor de $p$ & 0,325 & 0,407 & 0,020 & 0,830 & 0,290 \\
\hline \multicolumn{6}{|c|}{ Endoscópios digestivos/10.000 habitantes } \\
\hline Correlação de Pearson & $-0,058$ & $-0,099$ & 0,169 & 0,074 & $-0,097$ \\
\hline Valor de $\mathrm{p}$ & 0,750 & 0,476 & 0,034 & 0,259 & 0,342 \\
\hline \multicolumn{6}{|c|}{ Endoscópios digestivos disponíveis ao SUS/10.000 habitantes } \\
\hline Correlação de Pearson & 0,178 & 0,260 & 0,276 & 0,182 & $-0,008$ \\
\hline Valor de $\mathrm{p}$ & 0,355 & 0,062 & 0,001 & 0,006 & 0,939 \\
\hline \multicolumn{6}{|c|}{ Mamógrafos/10.000 habitantes } \\
\hline Correlação de Pearson & 0,186 & $-0,019$ & 0,123 & 0,165 & $-0,060$ \\
\hline Valor de $\mathrm{p}$ & 0,299 & 0,911 & 0,156 & 0,012 & 0,568 \\
\hline \multicolumn{6}{|c|}{ Mamógrafos disponíveis ao SUS/10.000 habitantes } \\
\hline Correlação de Pearson & 0,313 & 0,119 & 0,224 & 0,215 & $-0,009$ \\
\hline Valor de $\mathrm{p}$ & 0,087 & 0,498 & 0,014 & 0,002 & 0,931 \\
\hline
\end{tabular}

(continua) 
Tabela 3 (continuação)

\begin{tabular}{|c|c|c|c|c|c|}
\hline \multirow[t]{2}{*}{ Variável } & \multicolumn{5}{|c|}{ Regiões } \\
\hline & $\begin{array}{l}\text { Centro-oeste } \\
\qquad(n=35)\end{array}$ & $\begin{array}{l}\text { Norte } \\
(n=63)\end{array}$ & $\begin{array}{l}\text { Nordeste } \\
(n=174)\end{array}$ & $\begin{array}{l}\text { Sudeste } \\
(n=241)\end{array}$ & $\begin{array}{c}\text { Sul } \\
(n=101)\end{array}$ \\
\hline \multicolumn{6}{|c|}{ Equipamentos de radiologia médica total/10.000 habitantes } \\
\hline Correlação de Pearson & $-0,017$ & $-0,257$ & 0,004 & $-0,044$ & $-0,266$ \\
\hline Valor de $\mathrm{p}$ & 0,923 & 0,044 & 0,957 & 0,492 & 0,007 \\
\hline \multicolumn{6}{|c|}{ Equipamentos de radiologia médica disponíveis ao SUS/10.000 habitantes } \\
\hline Correlação de Pearson & 0,118 & 0,025 & 0,187 & 0,111 & $-0,120$ \\
\hline Valor de $\mathrm{p}$ & 0,501 & 0,846 & 0,014 & 0,084 & 0,232 \\
\hline \multicolumn{6}{|c|}{ Equipamentos de ultrassonografia/10.000 habitantes } \\
\hline Correlação de Pearson & 0,039 & $-0,170$ & 0,056 & 0,115 & $-0,076$ \\
\hline Valor de $\mathrm{p}$ & 0,823 & 0,184 & 0,467 & 0,076 & 0,448 \\
\hline \multicolumn{6}{|c|}{ Equipamentos de ultrassonografia disponíveis ao SUS/10.000 habitantes } \\
\hline Correlação de Pearson & 0,135 & $-0,017$ & 0,153 & 0,218 & $-0,082$ \\
\hline Valor de $\mathrm{p}$ & 0,441 & 0,893 & 0,044 & 0,001 & 0,415 \\
\hline
\end{tabular}

ECG: Eletrocardiograma; ESF: Estratégia Saúde da Família; IDH-M: Índice de Desenvolvimento Humano Municipal; SUS: Sistema Único de Saúde.

Nota: coeficiente de correlação de Pearson ( $r$ ) e valor de $p$ calculados a partir do IBM SPSS (IBM Corp., Armonk, Estados Unidos). Correlação fraca ( $r \geq 0$ e $\leq$ $0,3)$, correlação moderada $(r \geq 0,3$ e $\leq 0,6)$ e correlação forte $(r \geq 0,6$ e $\leq 0,9)$.

Tabela 4

Regressão linear múltipla entre proporção de internações por condições sensíveis à atenção primária e variáveis associadas, segundo regiões. Brasil, 2012.

\begin{tabular}{|c|c|c|c|}
\hline Região/Variável & Coeficiente (B) & Valor de p & $\mathbf{R}^{2}(\%)$ \\
\hline Região Norte & & & 29,1 \\
\hline Constante & 78,64 & 0,001 & \\
\hline IDH-M & $-84,66$ & 0,012 & \\
\hline Região Nordeste & & & 39,4 \\
\hline Constante & 13,52 & 0,000 & \\
\hline Total de médicos por 1.000 habitantes & $-4,61$ & 0,000 & \\
\hline Total de leitos por 1.000 habitantes & 2,61 & 0,000 & \\
\hline Equipamentos de radiologia médica disponíveis ao SUS por 10.000 habitantes & 5,75 & 0,000 & \\
\hline Região Sudeste & & & 23,2 \\
\hline Constante & 32,77 & 0,000 & \\
\hline Proporção da população com 60 anos ou mais & 0,862 & 0,000 & \\
\hline IDH-M & $-35,96$ & 0,003 & \\
\hline Total de leitos por 1.000 habitantes & $-0,467$ & 0,016 & \\
\hline ECG disponíveis ao SUS por 10.000 habitantes & $-2,26$ & 0,010 & \\
\hline Equipamentos de ultrassonografia disponíveis ao SUS por 10.000 habitantes & 2,50 & 0,019 & \\
\hline Proporção de leitos SUS & 0,039 & 0,043 & \\
\hline Região Sul & & & 23,0 \\
\hline Constante & 60,49 & 0,000 & \\
\hline IDH-M & $-65,28$ & 0,000 & \\
\hline Proporção da população com 60 anos ou mais & 0,604 & 0,019 & \\
\hline
\end{tabular}

ECG: eletrocardiograma; IDH-M: Índice de Desenvolvimento Humano Municipal; SUS: Sistema Único de Saúde.

Nota: coeficiente (B), R2 e valor de p calculados a partir do IBM SPSS (IBM Corp., Armonk, Estados Unidos). 
como na análise de correlação, na qual não houve associação estatisticamente significativa, nenhuma variável independente pode ser inserida na equação de regressão linear. Assim, a Tabela 4 expõe os resultados da regressão linear para quatro regiões (Norte, Nordeste, Sul e Sudeste).

No que concerne à Região Norte, dentre as variáveis que apresentaram correlação com o desfecho, a variável IDH-M é aquela com maior poder de explicação para a proporção de internações por condições sensíveis à atenção primária. O IDH-M consegue explicar, em média, 29,1\% da variação da proporção de internações por condições sensíveis à atenção primária nessa região, sendo que o aumento de uma unidade do IDHM está associado à redução de 84,6 pontos na proporção de internações por essas causas, mantidas as demais variáveis do modelo constantes.

Já na Região Nordeste, três variáveis se destacaram na regressão linear múltipla: o total de médicos por mil habitantes; o total de leitos por mil habitantes e equipamentos de radiologia médica disponíveis ao SUS por 10 mil habitantes. Juntas, essas três variáveis independentes conseguem explicar $39,4 \%$ da variação da proporção de internações por condições sensíveis à atenção primária na Região Nordeste. Dessas, a variável que melhor explica o desfecho nessa região é o total de médicos por mil habitantes. É possível observar a redução de 4,6 pontos na proporção das internações por condições sensíveis à atenção primária na região Nordeste a cada aumento de uma unidade no total de médicos por mil habitantes.

Na Região Sudeste, seis indicadores explicam significativamente o desfecho: proporção da população com 60 anos ou mais, IDH-M; total de leitos por mil habitantes; equipamentos de ECG disponíveis ao SUS por 10 mil habitantes; equipamentos de ultrassonografia disponíveis ao SUS por 10 mil habitantes e a proporção de leitos disponíveis ao SUS. Essas variáveis explicam, em média, $23,2 \%$ da variação da proporção das internações por condições sensíveis à atenção primária na Região Sudeste. Destaca-se a variável proporção da população com 60 anos como a que possui maior poder de explicação do desfecho na Região Sudeste. Além disso, os achados revelam a relevância do IDH-M na ocorrência das internações por condições sensíveis à atenção primária na Região Sudeste. O aumento de uma unidade dessa variável está associado a um decréscimo de 35,9 pontos na proporção dessas internações.

Por último, na Região Sul do país, duas variáveis se destacaram no modelo de regressão linear: IDH-M e a proporção da população idosa. Juntas, essas variáveis conseguem explicar 23\% da variação da proporção de internações por condições sensíveis à atenção primária nessa região. No entanto, assim como na Região Norte, o IDH-M é a variável com maior poder de explicação da proporção de internações por condições sensíveis à atenção primária na Região Sul. O aumento de uma unidade dessa variável provoca, em média, diminuição de 65,28 pontos na proporção de internações por condições sensíveis à atenção primária na Região Sul.

\section{Discussão}

A análise empreendida permitiu identificar um discreto declínio na proporção de internações por condições sensíveis à atenção primária no Brasil, particularmente de 2007 a 2012. No início do período analisado - 1998 a 2006 - em que pesem a expansão da ESF e os esforços de mudança do modelo de atenção no SUS, não houve queda expressiva nesse indicador no conjunto do país. Outras variáveis que potencialmente poderiam influenciar as internações por condições sensíveis à atenção primária também apresentaram alterações entre 1998 e 2012, em escala nacional, como a situação socioeconômica da população, a oferta de médicos e de leitos hospitalares.

Os resultados iniciais sugeriram a relevância de investigar diversas variáveis que poderiam influenciar as internações por condições sensíveis à atenção primária, além da cobertura pela ESF, em distintos contextos, dada a heterogeneidade regional do país. Nesse sentido, o presente estudo procurou explorar possíveis associações entre indicadores socioeconômicos, demográficos, de oferta pública e privada selecionados e as internações por condições sensíveis à atenção primária, em municípios maiores que 50 mil habitantes, das diferentes regiões do Brasil, no ano de 2012.

No que concerne ao modelo de atenção primária, pesquisas anteriores 15,27,28,29 apontaram que a expansão da ESF no Brasil favoreceu avanços em diversos indicadores de saúde, tais como melhoria no acesso à assistência à saúde; no manejo de patologias crônicas; captação e acompanhamento de gestantes no pré-natal; melhorias quanto às taxas de aleitamento materno, queda na mortalidade infantil (observados outros indicadores de desenvolvimento) e na mortalidade por doenças cardiovasculares. Macinko et al. 23 também destacam que a rápida expansão da estratégia pode ter, de fato, resultado em melhora no diagnóstico, no tratamento e acompanhamento dos pacientes, com destaque para os portadores de doenças crônicas, causando redução da necessidade de internação e, consequentemente, redução da proporção de internações 
por condições sensíveis à atenção primária. Por outro lado, estudos semelhantes realizados em distintas localidades do país 11,12,23 ressaltam que a redução na proporção de internações por condições sensíveis à atenção primária não foi homogênea, variando segundo grupos de causas, regiões, estados e porte municipal.

O presente estudo revelou que fatores relacionados às condições socioeconômicas, demográficas, à oferta de serviços e de profissionais médicos estiveram associados à proporção de internações por condições sensíveis à atenção primária em municípios com mais de 50 mil habitantes, no ano de 2012, de forma diferenciada entre as regiões do país.

Dentre as variáveis investigadas que mostraram correlação negativa com as internações por condições sensíveis à atenção primária, destacaram-se o IDH-M e o total de médicos por mil habitantes. Tal achado é semelhante aos resultados de pesquisa anterior, realizada no Reino Unido, por Giuffrida et al. 30, que apontam as características socioeconômicas e os fatores relacionados à oferta de serviços, tais como número de leitos e médicos generalistas, como principais fatores que explicam a variação de determinadas causas de internações por condições sensíveis à atenção primária, como diabetes e asma. Estudo recente realizado na Região Sul do Brasil por Nedel 21 também apontou a relevância das condições socioeconômicas nesse tipo de análise, uma vez que a probabilidade de internação por condições sensíveis à atenção primária foi maior que o dobro em indivíduos com pior situação econômica. Para o autor, tal achado sugere um efeito compensador do SUS sobre a iniquidade social, ainda mais evidente nas regiões cobertas pela ESF.

No que diz respeito à oferta de profissionais médicos, cabe mencionar que a distribuição desigual desses trabalhadores, com concentração nas capitais e grandes centros do país, é um problema estrutural do sistema de saúde brasileiro. Vários estudos destacam a importância da distribuição equitativa de profissionais de saúde, com destaque para médicos e enfermeiros, em todas as regiões do país, para a melhoria de diferentes indicadores de saúde, tais como os relacionados à mortalidade infantil e materna 31,32 . Nesse sentido, o presente estudo identificou uma associação entre uma maior disponibilidade de médicos por mil habitantes e a menor ocorrência de internações por condições sensíveis à atenção primária.

O problema de distribuição desigual de médicos tem sido enfrentado por meio de diferentes estratégias nas ultimas décadas, em geral de caráter fragmentado e temporário, tais como o Programa de Interiorização do Trabalho em Saú- de (PITS), o Programa de Educação pelo Trabalho em Saúde (PET-Saúde) e o Programa de Valorização do Profissional da Atenção Básica (PROVAB). Cabe salientar que esse foi um dos distintos problemas que afloraram com a expansão da ESF no Brasil. Conforme assinalam Campos \& Belisário 33, a carência de profissionais em termos quantitativos e qualitativos é um dos problemas mais graves evidenciados a partir da implantação da ESF. Além disso, o programa esbarrou em dificuldades de fixação dos médicos em diversas regiões e localidades do país, resultando em alta rotatividade desses profissionais, o que compromete a efetividade do modelo, pautado no vínculo entre profissionais e população atendida 34 . Para Campos et al. 35, tal fenômeno é fruto de um círculo vicioso, composto por más condições de trabalho, indefinições políticas, insegurança e demanda excessiva, que reforçam uma clínica reduzida ao núcleo profissional, dificultam o vínculo e facilitam a rotatividade profissional.

Na tentativa de enfrentar esse problema, o Ministério da Saúde lançou, em 2013, o programa Mais Médicos, que gerou polêmica na sociedade civil e no meio acadêmico por envolver a contratação temporária de médicos estrangeiros, sem revalidação do diploma, entre as ações para diminuir a carência de médicos nas periferias dos grandes centros e cidades do interior. Em que pese a polêmica em torno do programa, o seu caráter recente e a ausência de estudos de avaliação de sua efetividade até o momento, os resultados da presente pesquisa corroboram a importância da alocação adequada de médicos em todas as regiões do país, para buscar uma atenção primária resolutiva.

Cabe mencionar ainda que os achados deste estudo sugerem que a presença do profissional de saúde se faz mais importante do que a disponibilidade de equipamentos de apoio diagnóstico na redução das internações por condições sensíveis à atenção primária. Vale ressaltar, entretanto, que a prática de uma atenção primária abrangente engloba não só profissionais capacitados, mas suporte material e institucional, que inclui condições de trabalho satisfatórias e acesso a uma rede de atenção à saúde estruturada e hierarquizada.

O fato de os resultados não terem apontado associação significativa entre a cobertura populacional estimada pela ESF nos municípios e a menor proporção de internações por condições sensíveis à saúde primária na maioria das regiões suscitou a reflexão sobre a implantação do programa em municípios de médio e grande porte e a efetividade do modelo de atenção. De acordo com Campos ${ }^{36}$, para cumprir os preceitos expostos na política nacional de atenção primária, 
que orientam as ações de uma atenção primária abrangente e resolutiva, as equipes de atenção primária necessitam operar com três funções complementares: a clínica, a de saúde pública e o acolhimento (atendimento ao imprevisto e atenção à demanda). Segundo o autor, no Brasil, tem-se valorizado a saúde coletiva e subestimado as duas outras.

Outro aspecto investigado na pesquisa refere-se à proporção de beneficiários de planos de saúde. Apesar de sua expressão nos testes de correlação, tal variável não apresentou poder explicativo do desfecho em nenhuma região do país, quando foi aplicado o modelo de regressão linear. Tal achado possivelmente está relacionado às condições socioeconômicas, visto que os resultados sugerem alto poder explicativo e maior efeito da variável IDH-M sobre o desfecho.

Dentre as limitações do estudo, ressalte-se que foram incluídas somente as internações realizadas em unidades vinculadas ao SUS, excluindo-se, portanto, internações financiadas por meio de planos privados de saúde. Além disso, cabe destacar que foram avaliados somente os municípios com população acima de 50 mil habitantes, onde, em geral, a cobertura pela ESF é inferior aos demais municípios, fato, em parte, relacionado à existência de modelos concorrentes de APS e da atuação de seguimentos privados na assistência à saúde. Cabe mencionar ainda as dificuldades no acesso aos dados, bem como as características dos sistemas de informação utilizados, com destaque para o CNES, cujos dados sobre equipamentos só estão disponíveis a partir de 2005, e SIH-SUS, que não permite identificar casos de reinternações.

Em síntese, os resultados deste estudo sugerem que a redução das internações por condições sensíveis à atenção primária nas diferentes regiões do país está associada a diferentes fatores, com destaque para os relacionados às condições socioeconômicas e à oferta de serviços de saúde e de médicos. Tais achados contribuem para a reflexão crítica sobre as possibilidades e os limites da consolidação da ESF em grandes centros urbanos, bem como a necessidade de repensar as formas de organização da atenção primária nesses contextos específicos, incluindo análises sobre outros modelos.

Ressalta-se ainda que é importante a realização de novos estudos que incorporem períodos de análise mais longos ou que explorem, em maior detalhe, os fatores associados às internações por diferentes tipos de condições sensíveis à atenção primária, nos distintos cenários de configuração do sistema de saúde no país.

Não obstante os avanços alcançados a partir da expansão da ESF no Brasil, permanecem antigos desafios, tais como a distribuição mais adequada de médicos e outros profissionais de saúde no território nacional e a efetiva mudança do modelo de atenção, com melhoria da qualidade e da efetividade da atenção primária. Destacam-se ainda desafios que transcendem o setor saúde, como o investimento em infraestrutura e a redução das desigualdades sociais nas distintas regiões do país. 


\section{Resumen}

Las hospitalizaciones por condiciones sensibles a la atención primaria han sido utilizadas como un indicador de efectividad de la atención primaria. El artículo explora la asociación entre las variables seleccionadas y la proporción de condiciones sensibles a la atención primaria en Brasil. La investigación incluyó un análisis descriptivo de la evolución de indicadores nacionales de 1998 a 2012 y la realización de un estudio transversal de los municipios brasileños, con población de más de 50 mil habitantes, por región del país, para el año 2012, para el que se utilizaron técnicas estadísticas de correlación y regresión lineal. Los resultados muestran una discreta disminución en la proporción de condiciones sensibles a la atención primaria. Las condiciones socioeconómicas, demográficas y de oferta de médicos en los municipios mostraron una asociación con la proporción de condiciones sensibles a la atención primaria, con diferentes expresiones en las regiones del país. A pesar de los avances relacionados con la expansión de la Estrategia de Salud Familiar, persisten desafíos como la distribución adecuada de médicos y otros profesionales en el territorio nacional y el cambio del modelo de atención.

Atención Primaria de Salud; Hospitalización; Servicios de Salud; Estrategia de Salud Familiar

\section{Colaboradores}

Todas as autoras participaram igualmente do desenho do estudo, da análise dos dados, da concepção, redação e revisão final do artigo.

\section{Agradecimentos}

C. V. Machado e L. D. Lima são bolsistas de produtividade do CNPq. O desenvolvimento da pesquisa que originou o artigo conta com apoio financeiro do CNPq (Edital Universal 2013) e do Programa de Apoio à Pesquisa Desenvolvimento e Inovação da Ensp (Inova-Ensp).

\section{Referências}

1. Saltman RB, Rico A, Boerma W, editors. Primary care on the driver's seat? Organizational reform in European primary care. Berkshire: Open University Press; 2006. (European Observatory on Health Systems and Policies Series).

2. World Health Organization. The world health report 2008: primary health care now more than ever. http://www.who.int/whr/2008/whr08_en.pdf (acessado em 15/Jun/2014).

3. Kringos D, Boerma W, van der Zee J, Groenewegen P. Europe's strong primary care systems are linked to better population health but also to higher health spending. Health Aff (Millwood) 2013;

4. Atun RA. What are the advantages and the disadvantages of restructuring a health system to be more focused on primary care services? http:// www.euro.who.int/HEN/Syntheses/primaryvsspe cialist/20040115_15 (acessado em 15/Fev/2008).

5. Kringos DS, Boerma WG, Hutchinson A, van der Zee J, Groenewegen PP.The breadth of primary care: a systematic literature review of its core dimensions. BMC Health Serv Res 2010; 10:65.

6. Cueto $\mathrm{M}$. The origins of primary health care and selective primary health care. Am J Public Health 2004; 94:1864-74. 
7. Conill EM, Fausto MCR. Análisis de la problemática de la integración APS en el contexto actual: causas que inciden en la fragmentación de servicios y sus efectos en la cohesión social. Rio de Janeiro: Escola Nacional de Saúde Pública Sergio Arouca, Fundação Oswaldo Cruz; 2007. (Documento Técnico).

8. Giovanella L. Atenção primária à saúde seletiva ou abrangente? Cad Saúde Pública 2008; 24 Suppl 1:S21-3.

9. Organização Pan-Americana da Saúde/Organização Mundial da Saúde. Renovação da atenção primária nas Américas. Documento de posicionamento da Organização Pan-Americana de Saúde. http://www.opas.org.br/servico/arquivos/Sala 5575.pdf (acessado em 02/Ago/2007).

10. Billings J, Zeitel L, Lukomnik J, Carey TS, Blank AE, Newman L. Impact of socioeconomic status on hospital use in New York City. Health Aff (Millwood) 1993 ; 12:162-73.

11. Mafra F. O impacto da atenção básica em saúde em indicadores de internação hospitalar no Brasil [Dissertação de Mestrado]. Brasília: Faculdade de Economia, Administração, Contabilidade e Ciência da Informação e Documentação, Universidade de Brasília; 2010.

12. Boing AF, Vicenzi RB, Magajewski F, Boing AC, Moretti-Pires RO, Perez KG, et al. Redução das internações por condições sensíveis à atenção primária no Brasil entre 1998-2009. Rev Saúde Pública 2012; 46:359-66.

13. Carneiro JA, Caldeira AP, Santana KR. Percepção de médicos sobre a lista nacional de condições sensíveis à atenção primária. Rev APS 2011; 14:296-302.

14. Caetano R, Dain S. O Programa Saúde da Família e a reestruturação da atenção básica à saúde nos grandes centros urbanos: velhos problemas; novos desafios. Physis (Rio J.) 2002; 12:11-21.

15. Aquino R. Programa de Saúde da Família: determinantes e efeitos de sua implantação nos municípios brasileiros [Tese de Doutorado]. Salvador: Instituto de Saúde Coletiva, Universidade Federal da Bahia; 2006.

16. Escorel S, Giovanella L, Mendonça MHM, Magalhães R, Senna MCM. Avaliação da implementação do Programa Saúde da Família em dez grandes centros urbanos: síntese dos principais resultados. Brasília: Ministério da Saúde; 2002.

17. Escorel S, Giovanella L, Mendonça MHM, Senna MCM. O Programa de Saúde da Família e a construção de um novo modelo para a atenção básica no Brasil. Rev Panam Salud Pública 2007; 21:164-76.

18. Giovanella L, Mendonça MHM, Almeida PF, Escorel S, Senna MCM, Fausto MCR, et al. Saúde da família: limites e possibilidades para uma abordagem integral de atenção primária à saúde no Brasil. Ciênc Saúde Coletiva 2009; 4:783-94.

19. Ministério da Saúde. Portaria no 221, de 17 de abril de 2008. Diário Oficial da União 2008; 18 abr.
20. Alfradique ME, Bonolo PF, Dourado I, Lima-Costa MF, Macinko J, Mendonça CS, et al. Internações por condições sensíveis à atenção primária: a construção da lista brasileira como ferramenta para medir o desempenho do sistema de saúde (Projeto ICSAP - Brasil). Cad Saúde Pública 2009; 25:1337-49.

21. Nedel FB. Internações hospitalares evitáveis pela atenção primária: estudo do impacto do Programa de Saúde da Família sobre as internações por condições sensíveis à atenção primária no Rio Grande do Sul, Brasil [Tese de Doutorado]. Pelotas: Universidade Federal de Pelotas; 2009.

22. Guanais F, Macinko J. Primary care and avoidable hospitalizations: evidence from Brazil. J Ambul Care Manage 2009; 32:115-22.

23. Macinko J, de Oliveira VB, Turci MA, Guanais FC, Bonolo PF, Lima-Costa MF, et al. The influence of primary care and hospital supply on ambulatory care sensitive hospitalizations among adults in Brazil, 1999-2007. Am J Public Health 2011; 101:1963-70.

24. PROADESS. Matriz dos indicadores do Proadess. Internações por condições sensíveis à atenção primária. http://www.proadess.icict.fiocruz.br/ index.php?pag=fic\&cod=B11\&tab=1 (acessado em 20/Jun/2014)

25. Coordenação de Prevenção e Vigilância, Instituto Nacional de Câncer. Estimativa 2014: incidência de câncer no Brasil. http://www.inca.gov.br/esti mativa/2014/estimativa-24042014.pdf (acessado em 28/Jul/2014)

26. Levin J, Fox JA. Estatística para ciências humanas. 9a Ed. São Paulo: Pearson Prentice Hall; 2004.

27. Macinko J, Guanais FC, Souza MFM. Evaluation of the impact of the Family Health Program on infant mortality in Brazil, 1990-2002. J Epidemiol Community Health 2006; 60:13-9.

28. Macinko J, Marinho de Souza MF, Guanais FC, da Silva Simões CC. Going to scale with community-based primary care: an analysis of the family health program and infant mortality in Brazil, 1999-2004. Soc Sci Med 2007; 65:2070-80.

29. Rosella D, Harhay MO, Pompenet R, Aquino R, Barreto ML. Impact of primary health care on mortality from heart and cerebrovascular diseases in Brazil: a nationwide analysis of longitudinal data. BMJ 2014; 349:g4014.

30. Giuffrida A, Gravelle H, Roland M. Measuring quality of care with routine data: avoiding confusion between performance indicators and health outcomes. BMJ 1999; 319:94-8.

31. Sousa A, Dal Poz MR, Carvalho CL. Monitoring inequalities in the health workforce: the case study of Brazil 1991-2005. PLoS One 2012; 7:e33399.

32. Sousa A, Dal Poz MR, Carvalho CL. Reducing inequities in neonatal mortality through adequate supply of health workers: evidence from newborn health in Brazil. PLoS One 2013; 8:e74772. 
33. Campos FE, Belisário SA. O Programa Saúde da Família e os desafios para a formação profissional e a educação continuada. Interface Comun Saúde Educ 2001; 5:133-42.

34. Campos CVA, Malik AM. Satisfação no trabalho e rotatividade dos médicos do Programa de Saúde da Família. Rev Admin Pública 2008; 42:347-68.

35. Campos GW, Gutiérrez AC, Guerrero A, Cunha GT. Reflexões sobre a atenção básica e a estratégia saúde da família. In: Campos GW, Guerrero AVP, organizadores. Manual de práticas de atenção básica - saúde ampliada e compartilhada. São Paulo: Editora Hucitec; 2010. p. 121-42.
36. Campos GW. Reforma política e sanitária: a sustentabilidade do SUS em questão? Ciênc Saúde Coletiva 2007; 12:301-6.

Recebido em 19/Ago/2014

Versão final reapresentada em 26/Abr/2015

Aprovado em 21/Mai/2015 